\title{
Light scattering by coupled oriented dipoles: Decomposition of the scattering matrix
}

\author{
M. Ali Kuntman, ${ }^{1}$ Ertan Kuntman, ${ }^{2}$ Jordi Sancho-Parramon, ${ }^{3}$ and Oriol Arteaga ${ }^{2,4, *}$ \\ ${ }^{1}$ Independent researcher, Ankara, Turkey \\ ${ }^{2}$ Departament de Física Aplicada, IN2UB, Enphocamat Group, Universitat de Barcelona, C/ Martí i Franquès 1, Barcelona 08030, Spain \\ ${ }^{3}$ Rudjer Boskovic Institute, Bijenicka cesta 54, 10000, Zagreb, Croatia \\ ${ }^{4}$ LPICM, École Polytechnique, Université Paris-Saclay, 91128 Palaiseau, France
}

(Received 12 January 2018; published 11 July 2018)

\begin{abstract}
We study the optical response of two coupled oriented dipoles with the dimer axis perpendicular to the wave vector of light by analyzing how their scattering matrix can be decomposed. The scattering matrix can be written as a linear combination of three terms with a clear physical meaning: one for each particle and another that is responsible for the coupling and that vanishes for noninteracting or distant particles. We show that the interaction term may generate optical activity for certain scattering directions and that this effect manifests itself mostly in the near field. This simple and intuitive theory based on matrix and vector states of oriented dipoles also describes hybridization processes and Fano resonances. The decomposition method can be also formulated in terms of a hybrid basis that allows us to quantitatively determine the individual contribution of the in-phase and out-of-phase coupling modes to the overall intensity. Our method can help to understand the optical response of more complex nanostructures that can be decomposed into dipole terms. The results are illustrated in gold nanoantenna dimers which exhibit a strong dipolar resonance.
\end{abstract}

DOI: 10.1103/PhysRevB.98.045410

\section{INTRODUCTION}

Dipole interactions occur when two dipoles interact with each other through the space. For example dipolar interactions are responsible for electrostatic interactions inside molecules or between molecules which have permanent dipole(s): The partially negative portion of one polar molecule can be attracted to the partially positive portion of a second polar molecule. Dipole interactions are also very important for optical interactions in nanoscale particles. Since the oscillating electric field of a light wave acts on the charges of a particle, causing them to oscillate at the same frequency, the particle becomes a small radiating dipole whose radiation is seen as scattered light.

In optics, the interaction between induced dipoles is usually treated considering their mutual interaction potential [1]. This approach has also been generalized for the calculation of the light scattering by arbitrarily shaped particles, as a numerical technique known as the coupled dipole method or discrete dipole approximation (DDA) [2-4]. The method is based on a finite volume discretization of the scattering object, in which each volume element is modeled by an oscillating electric dipole that acts as receiver and emitter of electromagnetic radiation. Each dipole of the collection interacts with all the others, so that the fields at each dipole are determined by the incident field and interactions among all the dipoles of the collection. Similar approaches can also be used to study radiative heat transfer in systems of multiple dipoles [5].

In this paper we study analytically the simpler but relevant case of interaction between two oriented dipoles that form a dipole dimer. Despite that there are in the literature several

*oarteaga@ub.edu methods (both analytic and numeric) to predict the optical response of coupled dipoles [2,6-9], usually these methods provide the overall optical response and do not permit us to distinguish the contribution of the individual dipoles from the coupling contribution. We propose a study of the dipole interaction based on a decomposition of the scattering matrix. In our model the two dipoles are excited in phase by the incident wave that drives the system and we consider that the polarizabilities of the two particles are fully anisotropic, i.e., they can only be polarized in a predefined direction, in contrast to the classical consideration of a dimer made of isotropic spherical particles [10-12]. The existence of well defined directions of polarization brings our optical scattering problem closer to real nanostructures or metamaterials, where the direction of polarization is given by the morphology and orientation of the objects. The simplest example perhaps is the interaction between two plasmonic nanoantennas (a plasmonic dimer). Dipolar nano-objects (e.g., nanoparticles, nanorods, etc.) are generally regarded as most elementary components to build more complex composite nanostructures [13-16]. They are also the simplest "plasmonic molecules" and the possibility of controlling and measuring their chiroptical properties has created a growing interest in the usage of plasmonic dimers in biological applications such as DNA based nanostructures [17] or intracellular localization [18].

Our decomposition shows that the scattering matrix of oriented dipole dimers can be written as a linear combination of three scattering matrix terms with a clear physical meaning: one for each particle and the remaining one for the interaction. Despite the simplicity of the theory, it allows us to understand subtle effects such as the emergence of optical activity in certain achiral dimer configurations. Our decomposition method also allows for an easy representation of 
hybridization processes and of Fano resonances in anisotropic plasmonic nanostructures. In particular we show that the decomposition can be also formulated in terms of a hybrid basis which allows us to determine the individual contribution of each hybrid mode to the overall intensity. Finally, we apply our decomposition method to study the scattering matrix of oriented gold nanoantenna dimers in the spectral region where they exhibit a strong dipolar resonance. With this method we can quantify the relative contribution of coupled modes in the nanoantenna dimer and analyze how the interaction modifies the optical properties of the individual nanoantennas.

\section{GENERAL FRAMEWORK}

The induced electric dipole moment vector, $\mathbf{p}$, on a particle is proportional to the corresponding incident electric field, $\mathbf{E}_{0}(\mathbf{r})$ :

$$
\mathbf{p}=\varepsilon \tilde{\boldsymbol{\alpha}} \mathbf{E}_{\mathbf{0}}(\mathbf{r}),
$$

where $\tilde{\boldsymbol{\alpha}}$ is the electric polarizability of the particle, and $\varepsilon$ is the permittivity of the medium where the dipole is located. When we put two particles close to each other we have to consider mutual interactions. In this case, each one of the dipoles will experience the induced field of the other dipole. This coupling effect can be taken into account to find the actual dipole of each particle as follows [14]:

$$
\begin{aligned}
& \mathbf{p}_{\mathbf{1}}=\tilde{\boldsymbol{\alpha}}_{\mathbf{1}} \varepsilon \mathbf{E}_{\mathbf{o}}\left(\mathbf{r}_{\mathbf{1}}\right)+\tilde{\boldsymbol{\alpha}}_{\mathbf{1}} k^{2} \overline{\overline{\mathbf{G}}}_{\mathbf{E}}\left(\mathbf{r}_{1}-\mathbf{r}_{2}\right) \cdot \mathbf{p}_{\mathbf{2}}, \\
& \mathbf{p}_{\mathbf{2}}=\tilde{\boldsymbol{\alpha}}_{\mathbf{2}} \varepsilon \mathbf{E}_{\mathbf{0}}\left(\mathbf{r}_{2}\right)+\tilde{\boldsymbol{\alpha}}_{\mathbf{2}} k^{2} \overline{\overline{\mathbf{G}}}_{\mathbf{E}}\left(\mathbf{r}_{2}-\mathbf{r}_{1}\right) \cdot \mathbf{p}_{\mathbf{1}},
\end{aligned}
$$

where $\overline{\mathbf{G}}_{\mathbf{E}}$ is the free-space electric dyadic Green's function, $k$ is the wave number, and $\tilde{\boldsymbol{\alpha}}_{1}, \tilde{\boldsymbol{\alpha}}_{\mathbf{2}}$ are the polarizability tensors of the associated particles. Explicit form of dyadic Green's function is given by

$$
\begin{aligned}
\overline{\overline{\mathbf{G}}}_{\mathbf{E}}(\mathbf{r}) \cdot \mathbf{p}= & {\left[\left(1+\frac{i}{k r}-\frac{1}{k^{2} r^{2}}\right) \mathbf{p}\right.} \\
& \left.+\left(-1-\frac{3 i}{k r}+\frac{3}{k^{2} r^{2}}\right)\left(\mathbf{u}_{\mathbf{r}} \cdot \mathbf{p}\right) \mathbf{u}_{\mathbf{r}}\right] g(r),
\end{aligned}
$$

where $\mathbf{u}_{\mathbf{r}}$ is the unit vector along $\mathbf{r}$ and $g(r)=e^{i k r} / 4 \pi r$. The notation can be simplified if we let:

$$
\begin{aligned}
& A(r) \equiv\left(1+\frac{i}{k r}-\frac{1}{k^{2} r^{2}}\right) g(r), \\
& B(r) \equiv\left(-1-\frac{3 i}{k r}+\frac{3}{k^{2} r^{2}}\right) g(r) .
\end{aligned}
$$

Thus,

$$
\overline{\overline{\mathbf{G}}}_{\mathbf{E}}(\mathbf{r}) \cdot \mathbf{p}=A(r) \mathbf{p}+B(r)\left(\mathbf{u}_{\mathbf{r}} \cdot \mathbf{p}\right) \mathbf{u}_{\mathbf{r}} .
$$

Now let us consider the scattered far field at an observation point $z$. The total scattered field can be considered as a sum of the fields due to the interacting dipoles.

$$
E_{\text {scat }, j}=\frac{k^{2}}{\varepsilon}\left[\left(\overline{\overline{\mathbf{G}}}_{E}\left(\mathbf{r}_{f}-\mathbf{r}_{1}\right) \cdot \mathbf{p}_{1}\right)_{j}+\left(\overline{\overline{\mathbf{G}}}_{E}\left(\mathbf{r}_{f}-\mathbf{r}_{2}\right) \cdot \mathbf{p}_{2}\right)_{j}\right],
$$

where $j=x, y, z$. Note, however, that the $z$ component vanishes for a far-field detector in the $z$ axis.

In the following we will show that, according to the abovementioned dipole-dipole interaction scheme, the total $2 \times 2$ scattering matrix (or Jones matrix) of the whole process can be written as a linear combination of three Jones matrices, two of them corresponding to the usual Jones matrices of noninteracting dipoles and the third one being the Jones matrix due to the interaction. The interaction Jones matrix is scaled by a factor which is a function of the distance between the interacting dipoles so that for distant dipoles this coupling term consistently vanishes.

\section{DECOMPOSITION OF THE SCATTERING MATRIX OF THE COUPLED DIPOLE SYSTEM}

Let us consider a coherent parallel combination of interacting dipoles as given in Fig. 1. $d$ is the distance between the dipoles, which are located in the same $z$ plane, and are excited simultaneously by a plane wave. According to the figure the unit vectors $\mathbf{u}\left(\mathbf{r}_{2}-\mathbf{r}_{1}\right)$ and $\mathbf{u}\left(\mathbf{r}_{1}-\mathbf{r}_{2}\right)$ will be

$$
\mathbf{u}\left(\mathbf{r}_{2}-\mathbf{r}_{1}\right)=(0,-1,0), \mathbf{u}\left(\mathbf{r}_{1}-\mathbf{r}_{2}\right)=(0,1,0) .
$$

We consider that the polarizability of the dipoles is fully anisotropic, i.e., they can only polarize along a certain direction. The polarizability tensor of each dipole, $\tilde{\alpha}_{1}$ and $\tilde{\alpha}_{2}$, will be:

$$
\tilde{\boldsymbol{\alpha}}_{i}=\alpha_{i}\left(\begin{array}{ll}
a_{i} & b_{i} \\
b_{i} & c_{i}
\end{array}\right)
$$

with $i=1,2 . \alpha_{1}$ are $\alpha_{2}$ are the Lorentzian polarizabilities of the dipoles and $\tilde{\boldsymbol{\alpha}}_{1}, \tilde{\boldsymbol{\alpha}}_{2}$ are given by the following rotation:

$$
\tilde{\boldsymbol{\alpha}}_{i}=\mathbf{R}\left(-\phi_{i}\right) \alpha_{i}\left(\begin{array}{ll}
1 & 0 \\
0 & 0
\end{array}\right) \mathbf{R}\left(\phi_{i}\right)
$$

where

$$
\mathbf{R}(\phi)=\left(\begin{array}{cc}
\cos \phi & \sin \phi \\
-\sin \phi & \cos \phi
\end{array}\right),
$$

and $\phi_{1}$ and $\phi_{2}$ are the rotation angles as defined in Fig. 1. Then

$$
\begin{aligned}
a_{i} & =\cos ^{2}\left(\phi_{i}\right), \\
b_{i} & =\cos \left(\phi_{i}\right) \sin \left(\phi_{i}\right), \\
c_{i} & =\sin ^{2}\left(\phi_{i}\right) .
\end{aligned}
$$

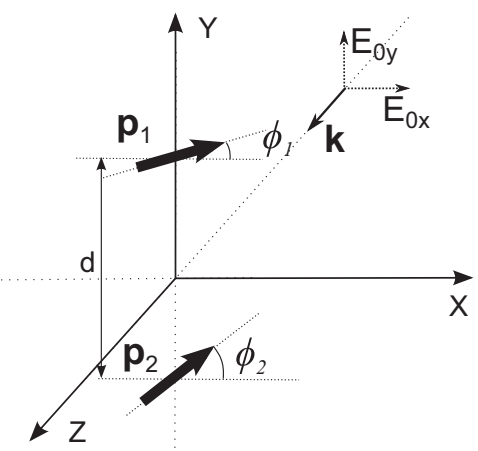

FIG. 1. Scheme of the geometry. Dimer axis and dipole vectors are perpendicular to the direction of propagation of incident light. 
From Eqs. (2) and (3) we can calculate the dipole vectors, whose components are given by:

$$
\begin{aligned}
& p_{1 x}=\varepsilon \alpha_{1} a_{1} E_{1 x}+\varepsilon \alpha_{1} b_{1} E_{1 y}+\alpha_{1} a_{1} \delta_{1} p_{2 x}+\alpha_{1} b_{1} \delta_{2} p_{2 y}, \\
& p_{1 y}=\varepsilon \alpha_{1} b_{1} E_{1 x}+\varepsilon \alpha_{1} c_{1} E_{1 y}+\alpha_{1} b_{1} \delta_{1} p_{2 x}+\alpha_{1} c_{1} \delta_{2} p_{2 y}, \\
& p_{2 x}=\varepsilon \alpha_{2} a_{2} E_{2 x}+\varepsilon \alpha_{2} b_{2} E_{2 y}+\alpha_{2} a_{2} \delta_{1} p_{1 x}+\alpha_{2} b_{2} \delta_{2} p_{1 y}, \\
& p_{2 y}=\varepsilon \alpha_{2} b_{2} E_{2 x}+\varepsilon \alpha_{2} c_{2} E_{2 y}+\alpha_{2} b_{2} \delta_{1} p_{1 x}+\alpha_{2} c_{2} \delta_{2} p_{1 y},
\end{aligned}
$$

where $k^{2} A \equiv \delta_{1}, k^{2}(A+B) \equiv \delta_{2}$. We can solve these coupled equations for the components of the dipole vectors:

$$
\begin{aligned}
& p_{1 x}=\varepsilon \alpha_{1} C_{1} \frac{\mathcal{E}_{1}+\alpha_{2} \Delta \mathcal{E}_{2}}{1-\alpha_{1} \alpha_{2} \Delta^{2}}, \\
& p_{1 y}=\varepsilon \alpha_{1} S_{1} \frac{\mathcal{E}_{1}+\alpha_{2} \Delta \mathcal{E}_{2}}{1-\alpha_{1} \alpha_{2} \Delta^{2}}, \\
& p_{2 x}=\varepsilon \alpha_{2} C_{2} \frac{\mathcal{E}_{2}+\alpha_{1} \Delta \mathcal{E}_{1}}{1-\alpha_{1} \alpha_{2} \Delta^{2}}, \\
& p_{2 y}=\varepsilon \alpha_{2} S_{2} \frac{\mathcal{E}_{2}+\alpha_{1} \Delta \mathcal{E}_{1}}{1-\alpha_{1} \alpha_{2} \Delta^{2}},
\end{aligned}
$$

where

$$
\begin{gathered}
C_{i}=\cos \phi_{i}, \quad S_{i}=\sin \phi_{i}, \\
\mathcal{E}_{i}=C_{i} E_{i x}+S_{i} E_{i y},
\end{gathered}
$$

and

$$
\Delta=C_{1} C_{2} \delta_{1}+S_{1} S_{2} \delta_{2} .
$$

Note that $\Delta$ is the only term that is related to coupling. A far field detection point, $z$, has characteristic vector $\mathbf{r}_{f}$ with a module much larger than those of the dipole vectors: $r_{f} \gg$ $r_{1}, r_{2}$. In these conditions $\mathbf{r}_{f}$ will be perpendicular to $\mathbf{r}_{1}$ and $\mathbf{r}_{2}$,

$$
\left(\overline{\overline{\mathbf{G}}}_{E}\left(\mathbf{r}_{f}-\mathbf{r}_{i}\right) \cdot \mathbf{p}_{i}\right)_{j}=\frac{e^{i k z}}{4 \pi z} p_{i j},
$$

where $i=1,2$ and $j=x, y$. If the detection point is equidistant to the dipoles we simply get:

$$
\begin{aligned}
& E_{\text {scatx }}=\beta\left(p_{1 x}+p_{2 x}\right), \\
& E_{\text {scaty }}=\beta\left(p_{1 y}+p_{2 y}\right),
\end{aligned}
$$

where

$$
\beta=\frac{k^{2} e^{i k z}}{4 \pi \varepsilon z} .
$$

According to Fig. 1, it is reasonable to assume that $\mathbf{E}_{1}\left(r_{1}\right)=$ $\mathbf{E}_{2}\left(r_{2}\right)=\mathbf{E}_{0}$ as it corresponds to a plane wave propagating in the $z$ direction. Then the scattering problem can be directly formulated with a $2 \times 2$ scattering matrix $\mathbf{T}$ that transforms the fields as follows:

$$
\left(\begin{array}{l}
E_{\text {scatx }} \\
E_{\text {scaty }}
\end{array}\right)=\left(\begin{array}{ll}
T_{11} & T_{12} \\
T_{21} & T_{22}
\end{array}\right)\left(\begin{array}{c}
E_{0 x} \\
E_{0 y}
\end{array}\right)=\left(\begin{array}{l}
T_{11} E_{0 x}+T_{12} E_{0 y} \\
T_{21} E_{0 x}+T_{22} E_{0 y}
\end{array}\right) .
$$

From Eqs. (22a) and (22b) we find that the $2 \times 2$ scattering matrix $\mathbf{T}$ can be presented as:

$$
\mathbf{T}=\gamma\left[\alpha_{1} \mathbf{J}_{1}+\alpha_{2} \mathbf{J}_{2}+\alpha_{1} \alpha_{2} \Delta \mathbf{J}_{\text {int }}\right],
$$

where $\mathbf{J}_{1}, \mathbf{J}_{2}$, and $\mathbf{J}_{\text {int }}$ are Jones matrices given by

$$
\begin{aligned}
\mathbf{J}_{i} & =\left(\begin{array}{cc}
C_{i}^{2} & C_{i} S_{i} \\
C_{i} S_{i} & S_{i}^{2}
\end{array}\right), \\
\mathbf{J}_{\text {int }} & =\left(\begin{array}{cc}
2 C_{1} C_{2} & C_{1} S_{2}+C_{2} S_{1} \\
C_{1} S_{2}+C_{2} S_{1} & 2 S_{1} S_{2}
\end{array}\right),
\end{aligned}
$$

and

$$
\gamma=\frac{\varepsilon \beta}{1-\alpha_{1} \alpha_{2} \Delta^{2}} .
$$

$\gamma$ can be understood as an overall (polarization independent) complex amplitude of scattering. Note that it is also affected by the coupling term $\Delta$.

In Eq. (25), $\mathbf{J}_{1}$ and $\mathbf{J}_{2}$ are the Jones matrices of the individual, noninteracting dipoles. As seen in Eq. (26), the scattering matrix of a horizontal $\left(\phi=0^{\circ}\right)$ or vertical $\left(\phi=90^{\circ}\right)$ dipolar particle is diagonal. $\mathbf{J}_{\text {int }}$ is a combined term that contributes only when there is interaction $(\Delta \neq 0)$. Therefore, whenever the dipoles are sufficiently separated the contribution of the interaction matrix $\mathbf{J}_{\text {int }}$ will be negligible. It is also possible that still for small separations between the dipoles the system has a vanishing $\Delta$ due to their particular orientations in the plane. This happens whenever the dipoles are orthogonal and the line joining the dipole centers is parallel to one of the dipole vectors as, for example, when $\phi_{1}=0^{\circ}$ and $\phi_{2}=90^{\circ}$ in Eq. (20). This situation will be discussed in more detail in Sec. III B.

Instead of using $2 \times 2$ scattering matrices it is sometimes useful to rearrange the information contained in the scattering matrix in a so-called four-component covariance vector. The covariance vector that corresponds to a Jones matrix is defined as follows:

$$
|h\rangle_{\mathbf{J}}=\frac{1}{2}\left(\begin{array}{c}
J_{11}+J_{22} \\
J_{11}-J_{22} \\
J_{12}+J_{21} \\
i\left(J_{12}-J_{21}\right)
\end{array}\right),
$$

where $J_{i j}$ are the elements of $2 \times 2$ the scattering matrix. We use the standard bra-ket notation of quantum mechanics, where the bra is the Hermitian conjugate of the ket and represented by a row vector. As it was discussed in Refs. $[19,20]$ the outer product $|h\rangle\langle h|$ generates a $4 \times 4$ covariance scattering matrix of rank 1 that can be considered as an analog of a pure state in quantum mechanics. In terms of covariance vectors we can, alternatively, write the decomposition in Eq. (25) as:

$$
|h\rangle=\gamma\left[\alpha_{1}|h\rangle_{1}+\alpha_{2}|h\rangle_{2}+\alpha_{1} \alpha_{2} \Delta|h\rangle_{\text {int }}\right],
$$

where:

$$
|h\rangle_{i}=\frac{1}{2}\left(\begin{array}{c}
1 \\
\cos ^{2}\left(\phi_{i}\right)-\sin ^{2}\left(\phi_{i}\right) \\
2 \sin \left(\phi_{i}\right) \cos \left(\phi_{i}\right) \\
0
\end{array}\right),
$$


and

$$
|h\rangle_{\text {int }}=\left(\begin{array}{c}
\cos \left(\phi_{1}-\phi_{2}\right) \\
\cos \left(\phi_{1}+\phi_{2}\right) \\
\sin \left(\phi_{1}+\phi_{2}\right) \\
0
\end{array}\right)
$$

Note that because the matrices in Eqs. (26) and (27) are symmetric, the fourth component of these covariance vectors is always zero. This reads as an absence of circular polarization effects [19,21].

\section{OPTICAL PROPERTIES OF DIMERS OF ANISOTROPIC PARTICLES}

The above presented decomposition of the scattering matrix or the covariance vector allows us to study several relevant optical properties characteristic of dipolar dimer systems. In particular, in the next subsections we will use our decomposition method to discuss circular polarization effects, hybrid modes, and Fano resonances. All these are well-known optical properties that have been previously analyzed in the context of dimer systems [6-11,13,22-27]. The advantage of the decomposition method that we have introduced is that it distinguishes the contribution of the individual dipoles from the coupling contribution and that works for dimers of anisotropic particles at any relative orientation in the plane, allowing, for example, the derivation of generalized analytic expressions for the dipole coupling that are a function of the orientation angles.

\section{A. Circular polarization effects}

Chiroptical effects with plasmonic dimers have been considered in several recent publications [28-31] as they are relatively easy to fabricate with modern techniques. The geometry considered in most of these works is different from Fig. 1, as they consider two misaligned dipoles in which the dimer axis is completely parallel to the wave vector of light. This is a chiral configuration that gives rise to optical activity in these plasmonic samples. In essence, this corresponds to the coupled oscillator model of Born and Kuhn (Born-Kuhn model) [31,32] which, when applied to a chiral geometry, is the basis for the classical theory of optical activity. This model provides an intuitive way to understand the generation of optical activity (circular dichroism and circular birefringence) in chiral media.

In general, the serial combination of two misaligned particles with a dipolar response leads to circular polarization effects, or chiroptical effects in the far-field detection. This can be intuitively seen by considering the Jones matrix product associated to a sequence of dipolar elements, e.g., $\mathbf{J}_{2} \mathbf{J}_{1}$, and transforming it into its associated covariance vector:

$$
|h\rangle_{\mathbf{J}_{2} \mathbf{J}_{1}}=\left(\begin{array}{c}
\cos \left(\phi_{1}-\phi_{2}\right) \\
\cos \left(\phi_{1}+\phi_{2}\right) \\
\sin \left(\phi_{1}+\phi_{2}\right) \\
i \sin \left(\phi_{1}-\phi_{2}\right)
\end{array}\right)
$$

in which the fourth component, associated to these circular effects or chiroptical effects [20,21], is nonvanishing. But this case of serial (sequential) combination of elements is totally different from the geometry given in Fig. 1. In Eqs. (31) and (32) the fourth components of the vectors were zero, which reads as an absence of circular effect as it could be expected from the achiral geometry of the problem.

However, depending on the location of the observation point, there may exist a varying phase difference between the radiation fields of the dipoles. Earlier, in Eqs. (22a) and (22b) we considered that both dipoles radiate to a point of the far field with the same complex factor $\beta$ [Eq. (23)], but if the detection point is not equidistant from the dipoles, we have to consider different phases.

$$
\begin{aligned}
& E_{\text {scatx }}=\beta p_{1 x}+\beta^{\prime} p_{2 x}, \\
& E_{\text {scaty }}=\beta p_{1 y}+\beta^{\prime} p_{2 y},
\end{aligned}
$$

where $\beta^{\prime}=\beta e^{i \chi} \cdot \chi$ is an additional phase term that accounts for the different optical paths from each dipole to the detector.

In this situation Eq. (25) must be replaced by

$$
\mathbf{T}=\gamma\left[\alpha_{1} \mathbf{J}_{1}+e^{i \chi} \alpha_{2} \mathbf{J}_{2}+\alpha_{1} \alpha_{2} \Delta \mathbf{J}_{\text {int }}^{\prime}\right],
$$

where

$$
\mathbf{J}_{\text {int }}^{\prime}=\left(\begin{array}{cc}
C_{1} C_{2}\left(1+e^{i \chi}\right) & C_{1} S_{2}+C_{2} S_{1} e^{i \chi} \\
C_{1} S_{2} e^{i \chi}+C_{2} S_{1} & S_{1} S_{2}\left(1+e^{i \chi}\right)
\end{array}\right) .
$$

Then the covariance vector associated with this interaction matrix is:

$$
|h\rangle_{\mathrm{int}}^{\prime}=\left(\begin{array}{c}
\cos \left(\phi_{1}-\phi_{2}\right)\left(1+e^{i \chi}\right) \\
\cos \left(\phi_{1}+\phi_{2}\right)\left(1+e^{i \chi}\right) \\
\sin \left(\phi_{1}+\phi_{2}\right)\left(1+e^{i \chi}\right) \\
-i \sin \left(\phi_{1}-\phi_{2}\right)\left(1-e^{i \chi}\right)
\end{array}\right)
$$

where the fourth component now is different from zero if the dipoles are not parallel to each other $\left(\phi_{1} \neq \phi_{2}\right)$. Note that when $\chi=\pi$ only the fourth component of $|h\rangle_{\text {int }}^{\prime}$ survives and the interaction term displays pure circular effects.

This emergence of chiroptical signals from achiral systems is an interferencelike phenomenon that arises when there is a phase (path) difference to the observation point. Translating the point of detection will also modify the values of optical activity signals, eventually also switching their signs. There are two other important aspects that need to be highlighted:

(i) The effect may manifest itself only for interacting dipoles. In noninteracting systems $(\Delta=0)$ with the geometry of Fig. 1, the superposition of the dephased dipolar scattering contributions is not sufficient to generate chiroptical effects in the scattering matrix. Note that the only matrix modified in Eq. (35) is the interaction matrix.

(ii) This effect is not observed in the far field unless large scattering angles are considered. When the detection distance is large compared to the separation of the dipoles $d$, the phase difference will be given by $\chi=2 \pi d x / \lambda D$ [33], where $D$ is the distance between the plane of the dipoles and the parallel plane that includes the detector, $\lambda$ is the wavelength, and $x$ is the position of the observation point along the axis parallel to the line connecting the two point dipoles. As in the far field $D \gg d$, this phase difference tends to be negligible in far field realizations unless one considers sufficiently large scattering angles (implying large $x$ ).

We believe that the progress in the near field microscopy offers new perspectives for exploiting this effect for biosensing. 

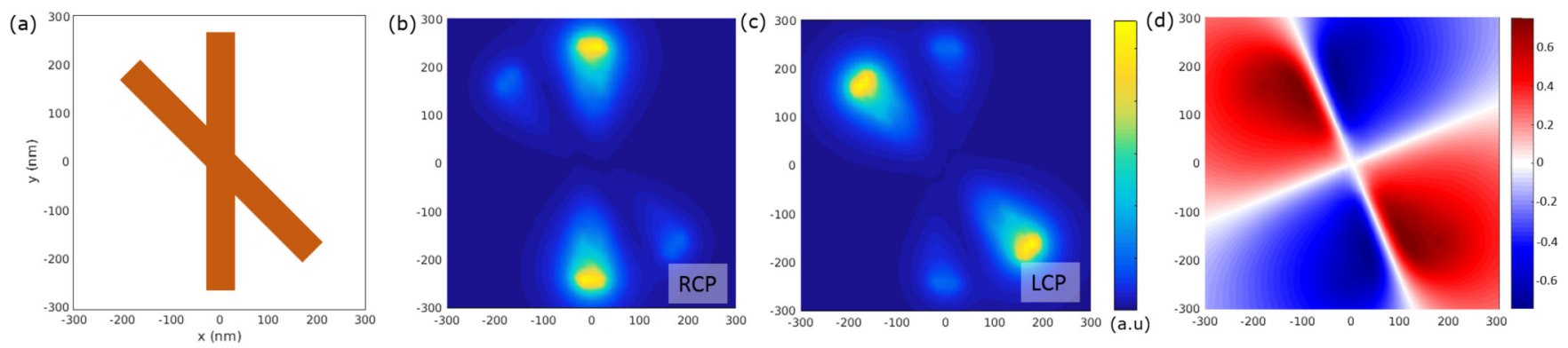

FIG. 2. Simulated response of two equal gold nanoantennas forming a cross by $45^{\circ}$. (a) shows the geometry and dimensions of the cross structure, which has a thickness of $50 \mathrm{~nm}$. (b) and (c) show the scattered field $|E|^{2}$ in a plane at $10 \mathrm{~nm}$ above the cross when the structure is illuminated with a plane wave of $1640 \mathrm{~nm}$ with right-and left-circular polarizations, respectively. In (d) we calculate the ratio $\left(I_{L C P}-I_{R C P}\right) /\left(I_{L C P}+I_{R C P}\right)$.

For example, this means that the near-field scattering fingerprint for such an arrangement of dipoles can be drastically modified by only adjusting the handedness of the incoming polarization, but without substantially affecting the far-field response. To illustrate this we have simulated the near field scattering of two crossed thin strips of gold with the nanoantenna geometry shown in Fig. 2(a). The simulation has been made with the boundary element method (BEM) [34,35] considering light with a wavelength that corresponds to the dipolar resonance of nanoantennas with this geometry (1640 $\mathrm{nm})$. The plane wave is polarized in the $x y$ plane and propagates along $+z$. The obvious differences between Fig. 2(b) and Fig. 2(c) show that the near field response of the structure (the figures show it in a plane $10 \mathrm{~nm}$ above the nanostructure) is strongly sensitive to the handedness of the incoming circular polarization, as it is anticipated by Eq. (37).

The ratio $\left(I_{L C P}-I_{R C P}\right) /\left(I_{L C P}+I_{R C P}\right)$, shown in Fig. 2(c), quantifies the differential scattering between left- and right-handed circular polarization. Note that it vanishes along two well defined orthogonal lines that cross the center of the nanostructure. These points correspond to zones that are equidistant from both nanoantennas, and there is no added phase $\chi(\chi=0)$. As we have previously pointed out, the strong circular effects [Fig. 2(c)] wash out in the far field because $\chi$ becomes negligible. In fact we already gave the far field response for this nanostructure in terms of the Mueller matrix in Ref. [19] and it was not sensitive to the handedness of the incoming polarization.

The emergence of chiroptical signals in certain systems with achiral geometries has been previously considered in molecules [36,37], crystals [38], and metamaterials $[39,40]$. Perhaps the most paradigmatic example is the water molecule [37] (point group $C_{2 v}$ ) where, due to the difference in electronegativity between the oxygen $(\mathrm{O})$ and hydrogen $(\mathrm{H})$ atoms, there is a dipole moment pointing from each $\mathrm{H}$ to the $\mathrm{O}$. These two permanent dipoles are misaligned by $104.45^{\circ}$ and they are both in the same plane, so it is the same type of geometry that we have considered. Our results can also be used to understand, at least qualitatively, the emergence of chiroptical signals in such planar systems for certain directions of observation. In fact optical activity in these achiral molecules and crystals is determined by an optical activity tensor, that when plotted, has the same twofold symmetry with alternating signs as the one displayed in Fig. 2(d).

\section{B. Hybrid modes}

Let us consider a coupling process as given in Fig. 1 . Equations (13) can be rearranged as follows:

$$
\begin{aligned}
& p_{1 x}-\alpha_{1} a_{1} \delta_{1} p_{2 x}-\alpha_{1} b_{1} \delta_{2} p_{2 y}=\varepsilon \alpha_{1} a_{1} E_{1 x}+\varepsilon \alpha_{1} b_{1} E_{1 y} \\
& p_{1 y}-\alpha_{1} b_{1} \delta_{1} p_{2 x}-\alpha_{1} c_{1} \delta_{2} p_{2 y}=\varepsilon \alpha_{1} b_{1} E_{1 x}+\varepsilon \alpha_{1} c_{1} E_{1 y} \\
& p_{2 x}-\alpha_{2} a_{2} \delta_{1} p_{1 x}-\alpha_{2} b_{2} \delta_{2} p_{1 y}=\varepsilon \alpha_{2} a_{2} E_{2 x}+\varepsilon \alpha_{2} b_{2} E_{2 y} \\
& p_{2 y}-\alpha_{2} b_{2} \delta_{1} p_{1 x}-\alpha_{2} c_{2} \delta_{2} p_{1 y}=\varepsilon \alpha_{2} b_{2} E_{2 x}+\varepsilon \alpha_{2} c_{2} E_{2 y} .
\end{aligned}
$$

Here we consider $p_{1 x}, p_{1 y}, p_{2 x}$, and $p_{2 y}$ as independent variables. Right hand side of the equations are the external fields, i.e., the driving forces of the dipoles, and we assume that the dipoles can be described as Lorentzian oscillators with a profile of the form:

$$
\alpha_{i}(\omega)=\frac{\eta_{i} \omega_{i}}{\omega_{i}^{2}-\omega^{2}-i \Gamma_{i} \omega},
$$

where $\omega$ is the frequency of the incoming radiation, $\omega_{i}$ is the frequency of resonance, $\eta_{i}$ is the amplitude of oscillation which depends on the particle size, and $\Gamma_{i}$ is the damping. For the analytical calculus of this section we will assume that there is no damping $\left(\Gamma_{i}=0\right)$.

The coupled Eqs. (38) can be written as a matrix equation:

$$
\mathbf{A P}=\mathbf{E},
$$

where $\mathbf{P}$ is a four-dimensional vector consisting of the components of the dipole vectors $\mathbf{p}_{1}$ and $\mathbf{p}_{2}, \mathbf{E}$ is also a fourdimensional vector associated with the right hand side of the coupled equations, and $\mathbf{A}$ is the following matrix:

$$
\mathbf{A}=\left(\begin{array}{cccc}
\lambda_{1} & 0 & -\delta_{1} a_{1} & -\delta_{2} b_{1} \\
0 & \lambda_{1} & -\delta_{1} b_{1} & -\delta_{2} c_{1} \\
-\delta_{1} a_{2} & -\delta_{2} b_{2} & \lambda_{2} & 0 \\
-\delta_{1} b_{2} & -\delta_{2} c_{2} & 0 & \lambda_{2}
\end{array}\right),
$$

where $\lambda_{1}=1 / \alpha_{1}, \lambda_{2}=1 / \alpha_{2}$. This problem can be treated as the well known problem of coupled mechanical (harmonic) oscillators [41]. Here the components of the electric dipole 
vectors play the role of position coordinates and the external fields are associated with the driving forces. Normal modes of the coupled system of oscillating dipoles can be found by equating the determinant of matrix $\mathbf{A}$ to zero, which leads to the equation:

$$
\lambda_{1}^{2} \lambda_{2}^{2}-\Delta^{2} \lambda_{1} \lambda_{2}=0
$$

where $\Delta$ is defined in Eq. (20).

There are four roots. Two of them are trivially given by $\lambda_{1} \lambda_{2}=0$, i.e., either $\lambda_{1}$ or $\lambda_{2}$ is zero. Nonzero roots are

$$
\lambda_{1} \lambda_{2}=\Delta^{2} \text {. }
$$

Note that this is also the condition that makes the denominator of $\gamma$ [Eq. (28)] vanish, so that there is a resonance in the scattering [Eq. (25)]. We can analytically examine the conditions for this resonance if the Lorentzian profile given in Eq. (39) (without damping) is assumed for the polarizabilities:

$$
\lambda_{1} \lambda_{2}=\left(\frac{\omega_{1}^{2}-\omega^{2}}{\eta_{1} \omega_{1}^{2}}\right)\left(\frac{\omega_{2}^{2}-\omega^{2}}{\eta_{2} \omega_{2}^{2}}\right)=\Delta^{2} .
$$

We solve now for $\omega$. Roots with $\lambda_{1} \lambda_{2}=0$ give $\omega=\omega_{1}$ and $\omega=\omega_{2}$ that correspond to trivial cases with no interaction between the dipoles. Nonzero roots give the frequencies for two hybridized modes:

$$
\omega^{ \pm}=\sqrt{\frac{\omega_{1}^{2}+\omega_{2}^{2} \pm \sqrt{\left(\omega_{1}^{2}-\omega_{2}^{2}\right)^{2}+4 \omega_{1}^{2} \omega_{2}^{2} \eta_{1} \eta_{2} \Delta^{2}}}{2}} .
$$

If the dipoles are identical $\left(\alpha_{1}=\alpha_{2}\right)$ we have

$$
\omega^{ \pm}=\omega_{0} \sqrt{1 \pm \eta \Delta} .
$$

For any pair of angles, $\phi_{1}$ and $\phi_{2}$, we always have two hybrid modes. For example, if we choose $\phi_{1}=\phi_{2}=0$

$$
\omega^{ \pm}=\omega_{0} \sqrt{1 \pm \eta \delta_{1}},
$$

if we choose $\phi_{1}=\phi_{2}=\frac{\pi}{2}$

$$
\omega^{ \pm}=\omega_{0} \sqrt{1 \pm \eta \delta_{2}} .
$$

The strength of the coupling may be evaluated with the aid of the parameter

$$
\omega_{c c}=\sqrt{\eta_{1} \eta_{2} \omega_{1} \omega_{2} \Delta^{2}}
$$

If the coupling is weak $\left(\omega_{c c} \ll\left|\omega_{1}-\omega_{2}\right|\right)$, the solutions of Eq. (45) reduce to

$$
\begin{aligned}
& \omega^{+} \approx \omega_{1}+\frac{\omega_{c c}^{2}}{4\left(\omega_{1}-\omega_{2}\right)}, \\
& \omega^{-} \approx \omega_{2}-\frac{\omega_{c c}^{2}}{4\left(\omega_{1}-\omega_{2}\right)},
\end{aligned}
$$

and in the case of strong coupling $\left(\omega_{c c} \gg\left|\omega_{1}-\omega_{2}\right|\right)$, the approximate solutions are

$$
\omega^{ \pm} \approx \frac{\omega_{1}+\omega_{2}}{2} \pm \frac{\omega_{c c}}{2}
$$

These formulas coincide with the resonances predicted by the plasmon hybridization model for two plasmonic particles given in Ref. [42], which the advantage that here we know how the coupling parameter $\omega_{c c}$ varies with the dipole orientation.
Figure 3 shows the switch of the energy of resonance for two coupled dipoles with equal polarizabilities $\left(\alpha_{1}=\alpha_{2}\right)$ as a function of the distance between them for six different geometrical arrangements. Their hybridization can be evaluated with Eq. (46). In both, $a\left(\phi_{1}=\phi_{2}=0^{\circ}\right)$ and $b\left(\phi_{1}=\phi_{2}=90^{\circ}\right)$, particles oscillate in phase but $a$ corresponds to a $\pi$-type stacking (dimer axis perpendicular to the dipole direction), while $b$ is a $\sigma$-type stacking (dimer axis parallel to the dipole direction). In this case the shift in energy is stronger than in $a$ and the resonance evolves to lower frequencies. Note that for $a$ and $b$, the incoming plane wave can only excite one of the two hybrid modes, the in-phase mode. The energy splitting of the in-phase and out-of-phase modes for these two cases are schematically shown in Fig. 4. The scattering matrices corresponding to these two cases (assuming the more general case $\alpha_{1} \neq \alpha_{2}$ ) are:

(i) Case (a), dipoles perpendicular to the dimer axis:

$$
\mathbf{T}_{a}=\varepsilon \beta \frac{\alpha_{1}+\alpha_{2}+2 \alpha_{1} \alpha_{2} \delta_{1}}{1-\alpha_{1} \alpha_{2} \delta_{1}^{2}}\left(\begin{array}{ll}
1 & 0 \\
0 & 0
\end{array}\right),
$$

(ii) Case (b), dipoles parallel to the dimer axis:

$$
\mathbf{T}_{b}=\varepsilon \beta \frac{\alpha_{1}+\alpha_{2}+2 \alpha_{1} \alpha_{2} \delta_{2}}{1-\alpha_{1} \alpha_{2} \delta_{2}^{2}}\left(\begin{array}{ll}
0 & 0 \\
0 & 1
\end{array}\right) .
$$

If only the near-field contribution of $A$ and $B$ (i.e., only the $d^{-3}$ term) is retained in the coupling parameters $\delta_{1}$ and $\delta_{2}$, we have that $\delta_{1} \propto-1 / d^{3}$ and $\delta_{2} \propto 2 / d^{3}$, and we may define the polarizabilities of the coupled systems for cases (a) and (b), respectively, as follows:

$$
\begin{aligned}
\alpha_{a} & =\frac{\alpha_{1}+\alpha_{2}-2 \alpha_{1} \alpha_{2} / d^{3}}{1-\alpha_{1} \alpha_{2} / d^{6}}, \\
\alpha_{b} & =\frac{\alpha_{1}+\alpha_{2}+4 \alpha_{1} \alpha_{2} / d^{3}}{1-4 \alpha_{1} \alpha_{2} / d^{6}},
\end{aligned}
$$

that respectively correspond to $\pi$ and $\sigma$ type bonding/ antibonding. The polarizabilites for the coupled dimer system were first defined in Ref. [43]. When $\alpha_{1}=\alpha_{2} \equiv \alpha$ and if only the near-field contributions of $\delta_{1}$ and $\delta_{2}$ are considered, far field scattering matrices given by Eqs. (53) and (54) simplify to:

$$
\begin{aligned}
& \mathbf{T}_{a}=\varepsilon \beta \frac{2 \alpha}{1+\alpha / d^{3}}\left(\begin{array}{ll}
1 & 0 \\
0 & 0
\end{array}\right), \\
& \mathbf{T}_{b}=\varepsilon \beta \frac{2 \alpha}{1-2 \alpha / d^{3}}\left(\begin{array}{ll}
0 & 0 \\
0 & 1
\end{array}\right) .
\end{aligned}
$$

Therefore for $\mathbf{T}_{a}$ only the in-phase mode that corresponds to $\alpha / d^{3}=-1$ (antibonding configuration) can be excited, and the out-of-phase mode (bonding configuration) is dark. Meanwhile, for $\mathbf{T}_{b}$ the in-phase mode corresponds to $2 \alpha / d^{3}=$ 1 and the out-of-phase mode (antibonding configuration) is dark. Dark modes cannot be optically activated with a plane wave, since it always generates an in-phase situation. Indeed, an out-of-phase mode could be activated if the dipoles were not located in the same $z$ plane, so that the incident electric field is not the same for both dipoles [44]. Another possibility to excite the out-of-phase mode, even for dipoles located in the same $z$ plane, is using inhomogeneous excitation, such as focused radiation [45]. 
(a)

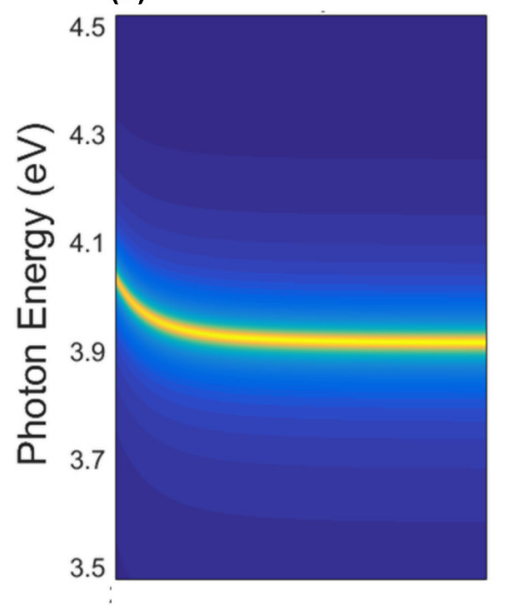

(d)

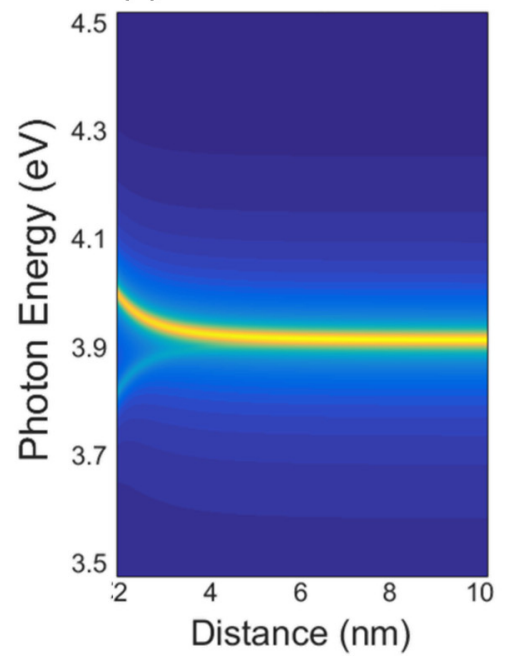

(b)
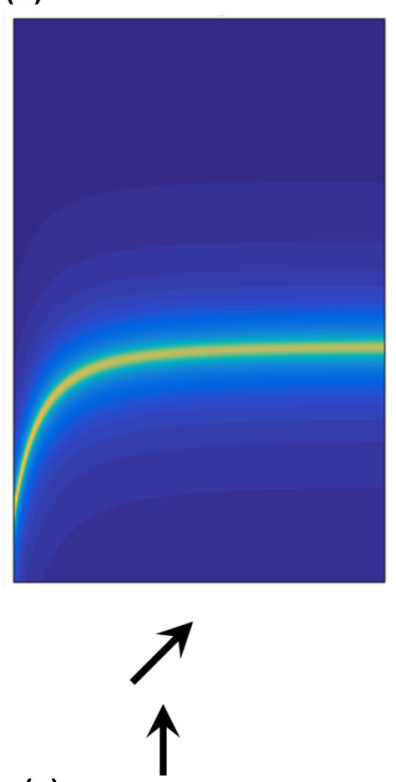

(e)

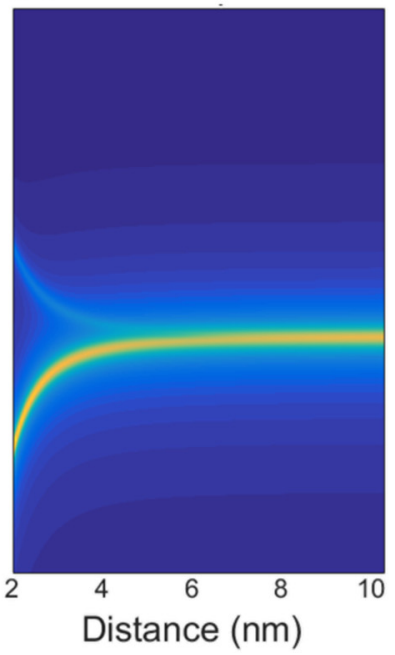

(c)
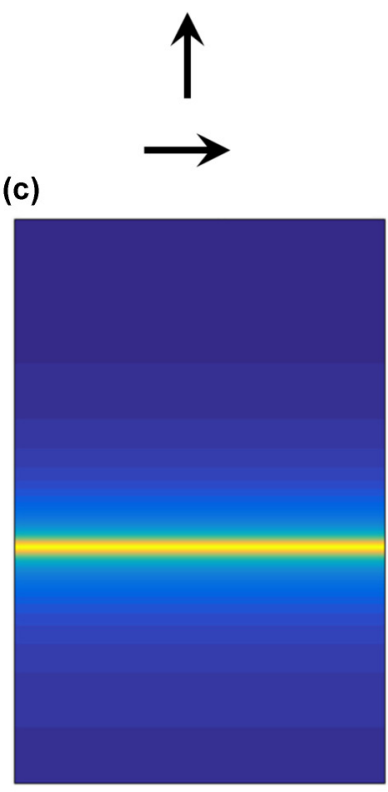

(f)

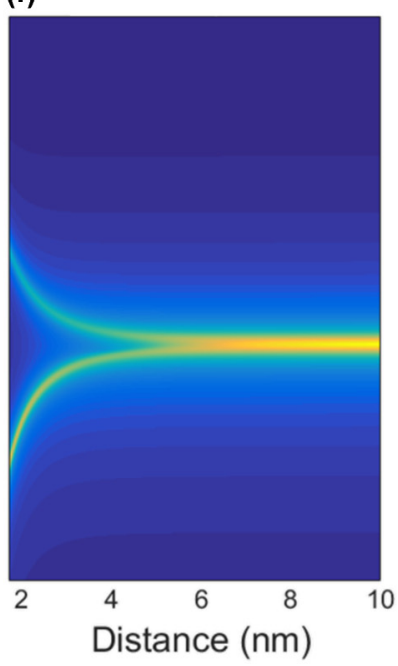

FIG. 3. Calculated intensity for the scattering of two coupled dipoles as a function of the distance between them. The dipoles can only polarize along the orientations shown by the arrows drawn at the top of each panel. These calculations correspond to illumination with a left-handed circularly polarized plane wave. The two particles were assumed to have polarizabilities of the same magnitude $\left(\alpha_{1}=\alpha_{2}\right)$ but different orientation. The spectroscopic values of the polarizability that we have used in this example are those that result from applying Clausius Mossotti relation to spherical silver particles in vacuum with a radii of $1 \mathrm{~nm}$ and using the Drude model of silver. Note that these spectroscopic values of polarizability are chosen for illustration purposes only and that the calculation is not describing a coupled system of spheres.

The remaining panels of Fig. 3 show misaligned dipoles. In (c) $\left(\phi_{1}=0^{\circ}\right.$ and $\left.\phi_{2}=90^{\circ}\right)$ there is no hybridization because $\Delta=0$ due to the orthogonality. Panels (d) $\left(\phi_{1}=0^{\circ}, \phi_{2}=45^{\circ}\right)$ and (e) $\left(\phi_{1}=90^{\circ}, \phi_{2}=45^{\circ}\right)$ show configurations which are, respectively, rather close to (a) and (b), but here both hybrid modes, in-phase and out-of-phase, can appear, despite that the in-phase is still much more intense than the other. In (f) $\left(\phi_{1}=-45^{\circ}, \phi_{2}=45^{\circ}\right)$ the directions of oscillation are orthogonal, like in (c), but here both particles have dipolar component parallel and perpendicular to the dimer axis and $\Delta$ is no longer vanishing. Note also that a rotation of the dipole arrows in (c) does not lead to the arrangement in (f). In this arrangement the two hybrid modes have a very similar intensity.

The availability of the scattering matrix together with the knowledge of the geometry of the problem provides very valuable information for the study of the hybridization. Suppose that we measure the transfer matrix of the whole system. Call 


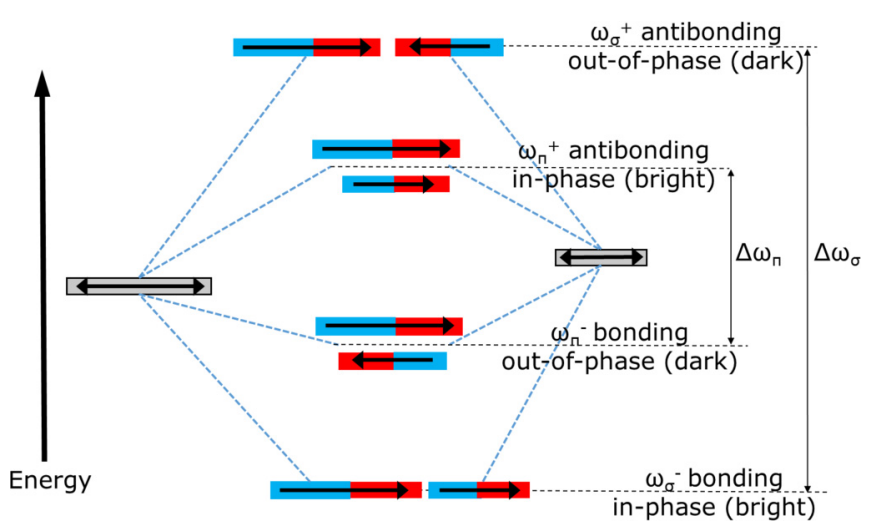

FIG. 4. Energy splitting in hybrid modes for a dipole dimer perpendicular ( $\pi$-type stacking) and parallel ( $\sigma$-type stacking) to the dimer axis. Red and blue colors, respectively, indicate positive and negative charge distribution.

this measured matrix, $\mathbf{T}_{m}$, and let the associated covariance vector be $|t\rangle_{m}$. If the orientation of the interacting dipoles with respect to the measurement coordinates are known then the matrices $\mathbf{J}_{1}, \mathbf{J}_{2}$, and $\mathbf{J}_{\text {int }}$ are also known [defined by Eqs. (26) and (27)], as well as their associated vectors $|h\rangle_{1},|h\rangle_{2}$, and $|h\rangle_{\text {int }}$. Therefore, we can either decompose $\mathbf{T}_{m}$ or $|t\rangle_{m}$ :

$$
\begin{aligned}
\mathbf{T}_{m} & =g_{1} \mathbf{J}_{1}+g_{2} \mathbf{J}_{2}+g_{\text {int }} \mathbf{J}_{\text {int }}, \\
|t\rangle_{m} & =g_{1}|h\rangle_{1}+g_{2}|h\rangle_{2}+g_{\text {int }}|h\rangle_{\text {int }},
\end{aligned}
$$

where $g_{1}, g_{2}$, and $g_{\text {int }}$ are complex amplitudes (expansion coefficients) that can be determined algebraically. Note that this three term decomposition of an interacting two-component system was already suggested in Ref. [19]. Comparing with Eq. (25) gives

$$
g_{1}=\gamma \alpha_{1}, \quad g_{2}=\gamma \alpha_{2}, \quad g_{\text {int }}=\gamma \alpha_{1} \alpha_{2} \Delta .
$$

Therefore,

$$
\frac{g_{\text {int }}^{2}}{g_{1} g_{2}}=\alpha_{1} \alpha_{2} \Delta^{2}
$$

We can then rewrite $\gamma$ in terms of the complex coefficients $g_{1}$, $g_{2}$, and $g_{\text {int }}$ :

$$
\gamma \propto \frac{1}{1-\left(\frac{g_{\text {int }}}{g_{1} g_{2}}\right)^{2}}=\frac{g_{1} g_{2}}{\left(\sqrt{g_{1} g_{2}}-g_{\text {int }}\right)\left(\sqrt{g_{1} g_{2}}+g_{\text {int }}\right)},
$$

where we can define

$$
v^{ \pm}=\sqrt{g_{1} g_{2}} \pm g_{\text {int }} .
$$

The maxima of $\gamma$ [i.e., the resonant conditions for the hybridized modes given by Eq. (43)] occur when either $v^{+}=0$ or $v^{-}=0$. Note that $v^{ \pm}$are in general complex numbers and their real and imaginary parts may not vanish simultaneously. We assume in our analysis of hybridization that $\operatorname{Re}\left(v^{ \pm}\right)=0$ is a condition of resonance if at the same time $\operatorname{Im}\left(v^{ \pm}\right)$is small or slowly varying.

We may now use $v^{+}$and $v^{-}$to define a new basis, $\left|h^{+}\right\rangle$and $\left|h^{-}\right\rangle$, in which $|t\rangle_{m}$ can be formulated as

$$
|t\rangle_{m}=v^{+}\left|h^{+}\right\rangle+v^{-}\left|h^{-}\right\rangle,
$$

where $|t\rangle_{m}$ is now written as a two term decomposition of hybrid modes, so that it is no longer necessary to make an explicit consideration of the interaction term. We may call $\left|h^{+}\right\rangle$ and $\left|h^{-}\right\rangle$the hybrid basis.

From direct comparison between Eqs. (57b), (62), and (61) we can find:

$$
\left|h^{ \pm}\right\rangle=\frac{g_{1}|h\rangle_{1}+g_{2}|h\rangle_{2}}{2 \sqrt{g_{1} g_{2}}} \pm \frac{|h\rangle_{\text {int }}}{2},
$$

where, as $g_{1}$ and $g_{2}$ can vary with frequency, the definition of the basis is frequency dependent. Note however that when $g_{1}=g_{2}$ the definition of the hybrid basis becomes merely geometrical and energy independent:

$$
\left|h^{ \pm}\right\rangle=\frac{|h\rangle_{1}+|h\rangle_{2}}{2} \pm \frac{|h\rangle_{\text {int }}}{2} .
$$

\section{Fano resonances}

Fano resonances in hybridized systems arise due to interference effects between the radiating states of the system [25,26]. We have shown that our interacting dimer system can be described as the superposition of three matrix or vector states. The most favorable condition for interference occurs when the superposed states are identical (fully overlapping), and this occurs when the states are characterized by the same normalized $2 \times 2$ scattering matrix or covariance vector.

Consider the case of dipoles parallel to the dimer axis, already presented in Eq. (54), which can be now written as

$$
\mathbf{T}_{b}=\gamma\left[\alpha_{1}\left(\begin{array}{ll}
0 & 0 \\
0 & 1
\end{array}\right)+\alpha_{2}\left(\begin{array}{ll}
0 & 0 \\
0 & 1
\end{array}\right)+2 \alpha_{1} \alpha_{2} \delta_{2}\left(\begin{array}{ll}
0 & 0 \\
0 & 1
\end{array}\right)\right],
$$

to highlight that $\mathbf{J}_{1}=\mathbf{J}_{2}=\mathbf{J}_{\text {int }}$. Alternatively, this can be also presented by a covariance vector:

$$
|h\rangle_{b}=\gamma\left(\alpha_{1}+\alpha_{2}+2 \alpha_{1} \alpha_{2} \delta_{2}\right)\left(\begin{array}{c}
1 / 2 \\
-1 / 2 \\
0 \\
0
\end{array}\right)
$$

The scattering intensity is given by

$$
\langle h \mid h\rangle_{b}=\left|\gamma\left(\alpha_{1}+\alpha_{2}+2 \alpha_{1} \alpha_{2} \delta_{2}\right)\right|^{2} / 2 .
$$

In the previous section we have shown that the denominator of $\gamma$ is a key to analyze the hybrid resonances, but $\gamma$ is an overall factor that is not taking into account interferences between the states. Interference takes place in the superposition term $\alpha_{1}+\alpha_{2}+2 \alpha_{1} \alpha_{2} \delta_{2}$ and, for example, one can expect a Fano dip when the real part of this superposition term vanishes and the imaginary part is small or slowly varying. This happens when there is destructive interference between the states. Figure 5 shows an example of this effect by considering the same calculus as in Fig. 3(b) but know with $\alpha_{1} \neq \alpha_{2}$. One can observe dips in the radiated intensity in between the two resonant modes.

Note that, in this configuration, no interference can occur if $\alpha_{1}=\alpha_{2} \equiv \alpha$ because then the scattering matrix simplifies to:

$$
\mathbf{T}_{b}=\frac{\varepsilon \beta \alpha}{1-\alpha \delta_{2}}\left(\begin{array}{ll}
0 & 0 \\
0 & 1
\end{array}\right)
$$

which does not allow interference because it contains just a single state. Indeed, symmetry breaking between coupling dipoles 

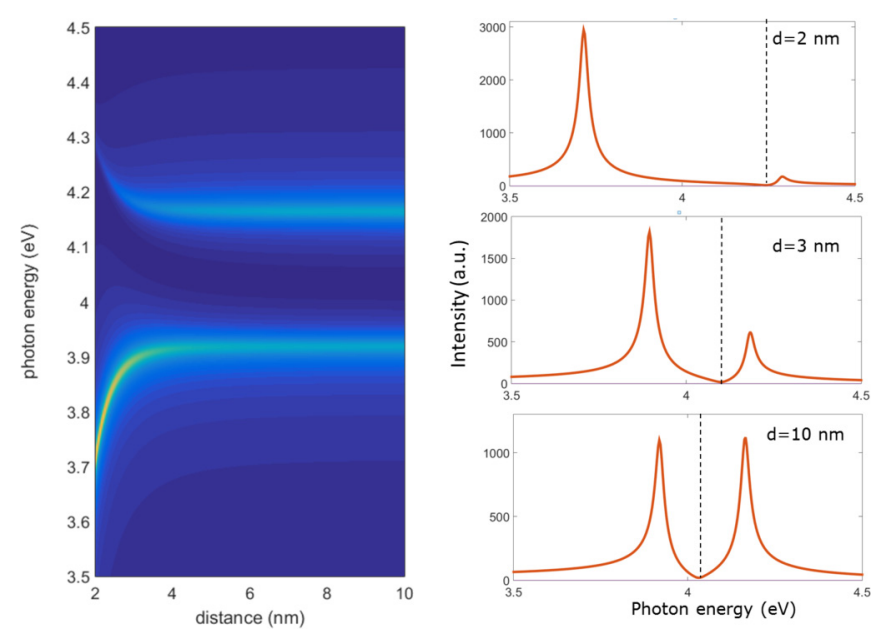

FIG. 5. Scattering intensity as a function of the distance for two parallel dipoles with different polarizabilities. Because of the interference, there is Fano dip (dashed line) in between the two resonant modes.

is a standard requirement for the generation of Fano resonances $[46,47]$. A more in-depth analysis of Fano resonances in other dimer configurations will be the subject of a future work.

\section{APPLICATION TO PLASMONIC NANOANTENNAS}

The analytic theory of light scattering by two coupled oriented dipoles that we have developed thus far can be useful to describe light-matter interaction processes which involve material structures that have dipolar responses. One good example is the case of plasmonic nanoantennas that we have already used in Sec. III A. Of particular interest is studying how the interaction affects the outcomes of optical measurements, since this analytic method may eventually permit us to distinguish the interaction contribution from the overall measured far-field optical response. With modern Mueller matrix polarimetry approaches it is possible to measure the complete scattering matrix in different plasmonic systems [48,49], even for single particles [50].

We apply this analytic method of the interaction to the far field response of a nanoantenna dimer made of gold and we analyze the same basic geometry as in Fig. 2, but for cases that differ in the distance between nanoantennas, as shown in Fig. 6(a). The far-field $2 \times 2$ Jones scattering matrix for these six configurations is calculated with the BEM method and then converted into a covariance vector to apply the decomposition of Eq. (57b).

The covariance vectors $|h\rangle_{1},|h\rangle_{2}$, and $|h\rangle_{\text {int }}$ are simply given by the geometry of the considered nanoantenna dimer. As in our example $\phi_{1}=90^{\circ}$ and $\phi_{2}=135^{\circ}$, the covariance vectors [defined in Eqs. (31) and (32)] will be:

$$
|h\rangle_{1}=\frac{1}{2}\left(\begin{array}{c}
1 \\
-1 \\
0 \\
0
\end{array}\right), \quad|h\rangle_{2}=\frac{1}{2}\left(\begin{array}{c}
1 \\
0 \\
-1 \\
0
\end{array}\right), \quad|h\rangle_{\text {int }}=\frac{1}{\sqrt{2}}\left(\begin{array}{c}
1 \\
-1 \\
-1 \\
0
\end{array}\right) .
$$
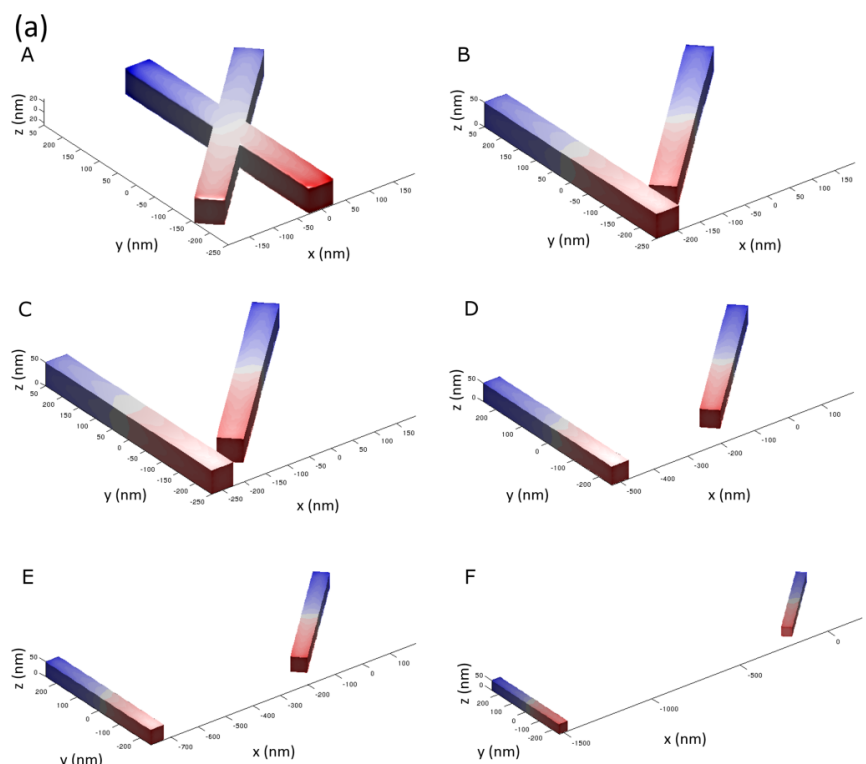

(b)

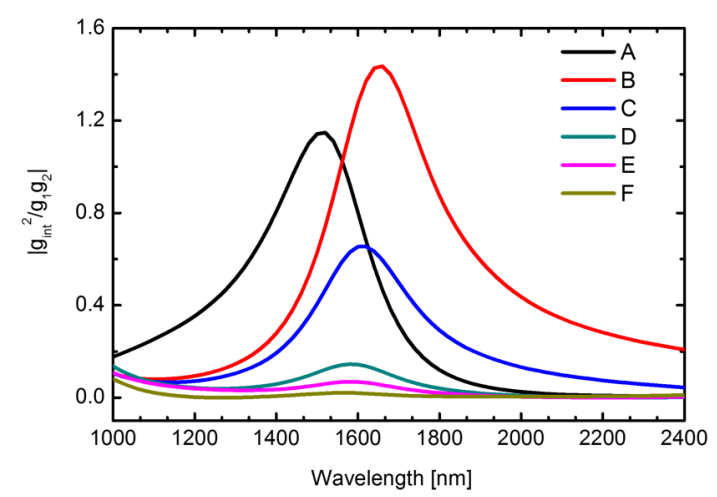

FIG. 6. (a) Different geometries of nanoantenna dimers considered in the BEM simulations. The only difference between the considered cases is the distance between the center of the nanoantennas $(d)$, which is respectively set at $0 \mathrm{~nm}, 225 \mathrm{~nm}, 250 \mathrm{~nm}, 500 \mathrm{~nm}, 750 \mathrm{~nm}$, and $1500 \mathrm{~nm}$ in A, B, C, D, E, and F. (b) Spectroscopic values of $\left|g_{\text {int }}^{2} / g_{1} g_{2}\right|$ for the six different configurations.

In this basis, Eq. (57b) leads to a system of three equations and three unknowns (note that the fourth equation is trivial because, for the present simulations of the nanoantennas, the fourth component of the covariance vector $|t\rangle_{m}$ is always zero), hence, it is possible to find $g_{1}, g_{2}$, and $g_{\text {int }}$ :

$$
\begin{aligned}
g_{1} & =2\left(h_{0}+h_{2}\right), \quad g_{2}=2\left(h_{0}+h_{1}\right), \\
g_{\text {int }} & =-\sqrt{2}\left(h_{0}+h_{1}+h_{2}\right),
\end{aligned}
$$

where $h_{0}, h_{1}$, and $h_{2}$ are, respectively, the first, second and third complex elements of the covariance vector $|t\rangle_{m}$ corresponding to the simulated scattering Jones matrix with the BEM method.

Therefore we can write:

$$
\frac{g_{\text {int }}^{2}}{g_{1} g_{2}}=\frac{\left(h_{0}+h_{1}+h_{2}\right)^{2}}{2\left(h_{0}+h_{1}\right)\left(h_{0}+h_{2}\right)} .
$$

The results of this analysis for the six cases presented in Fig. 6(a) are given in Fig. 6(b). We plot $\left|g_{\text {int }}^{2} / g_{1} g_{2}\right|$ (where the bars $|\ldots|$ denote the complex modulus) as a function of the wavelength. The most obvious result is that $\left|g_{\text {int }}^{2} / g_{1} g_{2}\right|$ diminishes as the distance between the nanoantennas increases. 


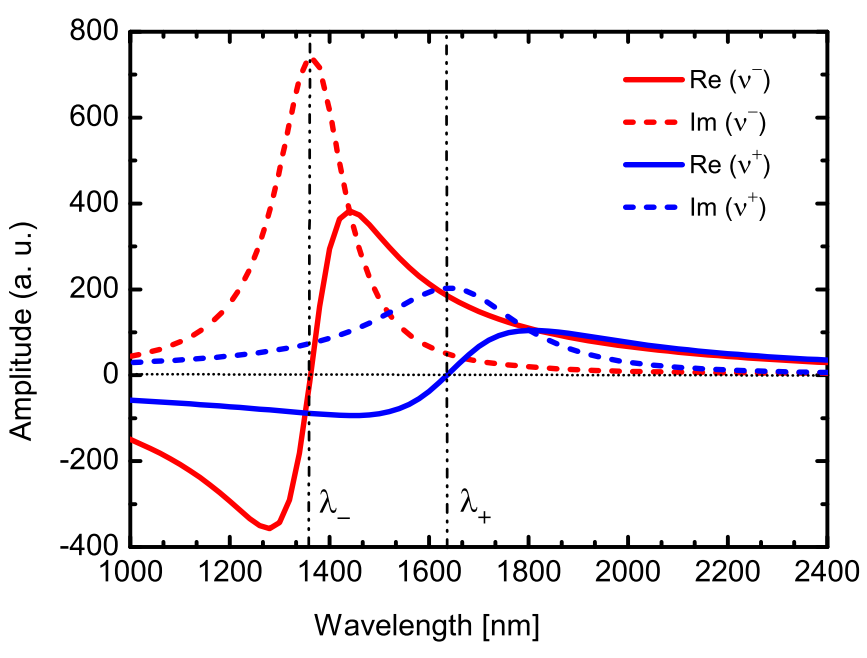

FIG. 7. Complex coefficients $v^{+}$and $v^{-}$corresponding to simulation A. The vertical lines indicate the position of the hybridization resonances.

Note that for cases $\mathrm{D}, \mathrm{E}$, and $\mathrm{F}\left|g_{\text {int }}^{2} / g_{1} g_{2}\right|$ is very small across all the spectrum, which indicates that interaction is weak. Only for cases A and B the interaction leads to a marked hybridization in the overall scattering intensity. For the remaining cases, hybridization mostly manifests itself as peak broadening, and the two hybrid contributions are not clearly distinguishable in the overall intensity unless $v^{+}$and $v^{-}$are calculated.

Hybridized frequencies can be calculated by analyzing the complex amplitudes $v^{+}$and $v^{-}$of the hybrid basis. As discussed earlier, the resonances appear when the real part of $\nu^{ \pm}$is zero and the imaginary part is small or slowly varying (i.e., maxima of gamma). This is illustrated in Fig. 7, which displays the spectroscopic values of $v^{+}$and $v^{-}$and the spectral position of the resonances for Simulation A. The resonant peaks found for all the simulated cases are summarized in Table I.

The values in Table I show that the hybrid frequencies are very sensitive to the distance between the nanoantennas. The spectral response can be therefore analyzed and designed by changing the interparticle distance, without need to modify the particle shape and orientation. This could be important for potential applications in near-field biosensing, in which small changes of distance translate in abrupt changes of light intensity. This is the basis of nanometrology tools such as plasmon rulers $[13,51,52]$. In fact our analytic approach allows

TABLE I. Spectral position of the in-phase $\left(\lambda_{+}\right)$and out-of-phase $\left(\lambda_{-}\right)$hybridization resonances for the considered cases.

\begin{tabular}{lccc}
\hline \hline Case & $\lambda_{+}(\mathrm{nm})$ & $\lambda_{-}(\mathrm{nm})$ & $\left|\lambda_{+}-\lambda_{-}\right|(\mathrm{nm})$ \\
\hline A & 1635 & 1360 & 275 \\
B & 1484 & 1828 & 344 \\
C & 1499 & 1692 & 193 \\
D & 1522 & 1620 & 98 \\
E & 1532 & 1614 & 82 \\
F & 1594 & 1545 & 49 \\
\hline \hline
\end{tabular}
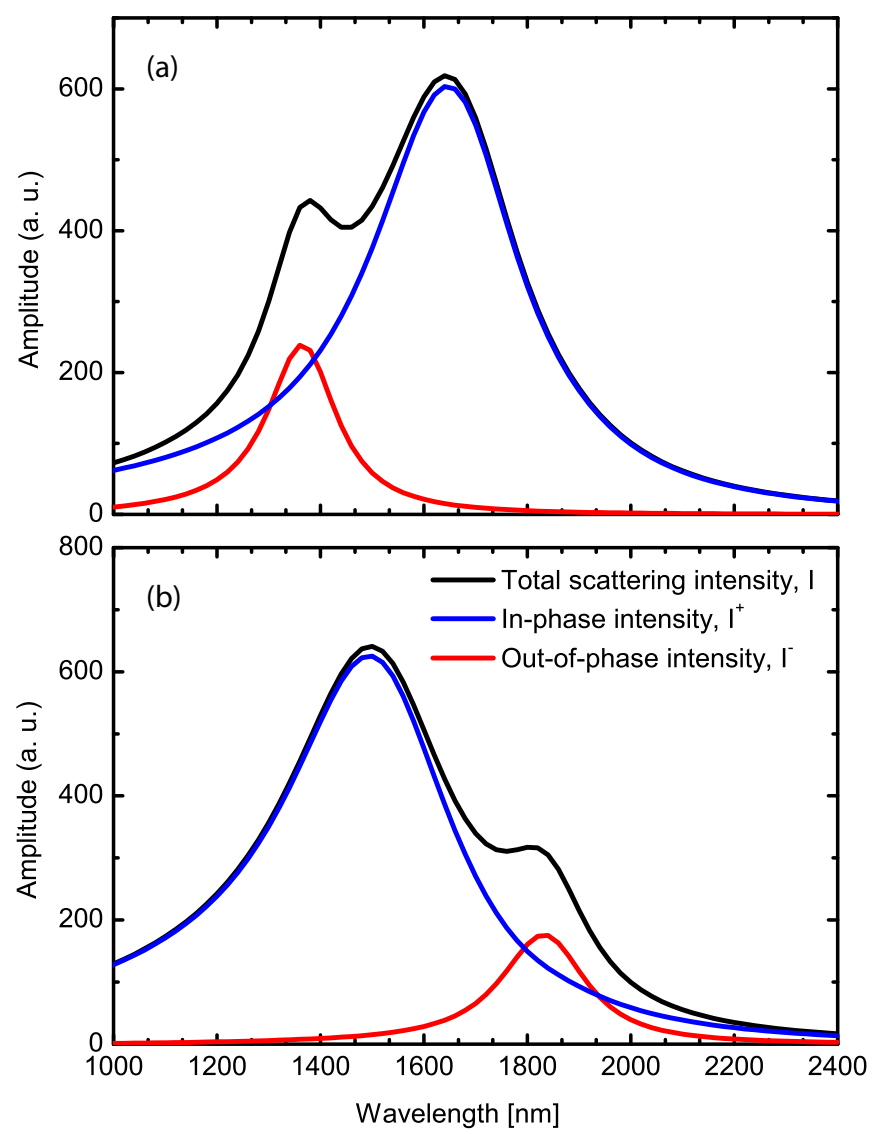

FIG. 8. Comparison of the in-phase and out-phase calculated intensities $\left(I^{+}\right.$and $I^{-}$, respectively) with respect to the overall simulated scattering intensity $(I)$ for simulations A (a) and B (b).

us to determine the position of the hybrid frequencies and study their intensity. The intensity of scattering for each hybrid mode is

$$
I^{ \pm}=v^{ \pm} v^{ \pm *}\left\langle h^{ \pm} \mid h^{ \pm}\right\rangle
$$

where * indicates the complex conjugate and

$$
\left|h^{ \pm}\right\rangle=\frac{1}{4 \sqrt{2}}\left(\begin{array}{c}
2 \sqrt{2} \pm 2 \\
-\sqrt{2} \pm 2 \\
-\sqrt{2} \pm 2 \\
0
\end{array}\right)
$$

corresponding to the basis in Eqs. (69). Note that for an orthogonal hybrid basis like this one $\left\langle h^{+} \mid h^{-}\right\rangle=0$. In fact the hybrid bases are orthogonal whenever $g_{1}=g_{2}$, and when this occurs:

$$
I=I^{+}+I^{-},
$$

where $I$ is the overall scattering intensity $(I=\langle h \mid h\rangle)$. Figure 8 shows how the decomposition in the hybrid basis clearly differentiates the contributions of the in-phase and out-of-phase modes to overall scattered intensity. Notably, the amplitudes $I^{+}$and $I^{-}$can perfectly account for the position in energy and amplitude of the in-phase and out-of-phase hybridized peaks. This shows the usefulness of the proposed three term decomposition method for dimer systems. 


\section{CONCLUSION}

We have shown that the scattering matrix of a coupled dipole dimer can be written as a linear combination of three states which have a clear physical (and geometrical) meaning. The study of the interaction term $\Delta \mathbf{J}_{\text {int }}$ and the complex factor $\gamma$ permits a clear understanding of phenomena occurring in some particle dimers, such as the emergence of optical activity in certain achiral configurations, hybridization effects, and Fano resonances. The application of the formalism has been illustrated by studying the dipolar resonance of coupled plasmonic nanoantennas which were simulated by elaborated numerical methods. Some of the results given by our analytic calculus are:

(i) The near-field scattering fingerprint for an achiral dipole dimer can be drastically modified by only adjusting the handedness of the incoming polarization, but this will not substantially alter far-field response for small scattering angles.

(ii) We have obtained an analytic expression [Eq. (45)] that provides the frequencies of the hybrid modes for any geometric arrangement of dipoles in a plane. Fano-like resonances can be also explained from the interference between the matrix states of our decomposition. (iii) We have shown that the hybridization-induced spectral splitting in coupled oriented dimers can be well explained by our decomposition method. The hybrid basis that we have defined allows us to quantitatively distinguish the contribution of the in-phase and out-of-phase modes to the overall scattering intensity in particles with anisotropic polarizability, something that, to our knowledge, has never been achieved with prior descriptions of plasmonic hybridization processes. This is possible even for weakly coupled particles, where no evident peak splitting is observed in the scattering cross sections.

Our analytical model provides a simple framework to understand and quantify the relative contribution of coupled modes in complex nanostructures. We think that this analytic method can be particularly useful in nanophotonic applications that make use of small antennalike elements for controlling electromagnetic waves such as optical trapping, single-molecule localization, and recognition or surface-enhanced spectroscopy.

\section{ACKNOWLEDGMENTS}

This work was partially funded by Ministerio de Economia y Competividad (EUIN2017-88598) and European Commission (Polarsense, MSCA-IF-2017-793774).
[1] J. D. Jackson, Classical electrodynamics (Wiley, India, 2011).

[2] E. M. Purcell and C. R. Pennypacker, Astrophys. J. 186, 705 (1973).

[3] S. B. Singham and C. F. Bohren, J. Opt. Soc. Am. A 5, 1867 (1988).

[4] B. T. Draine and P. J. Flatau, J. Opt. Soc. Am. A 11, 1491 (1994).

[5] R. Messina, M. Tschikin, S.-A. Biehs, and P. Ben-Abdallah, Phys. Rev. B 88, 104307 (2013).

[6] B. Rolly, B. Stout, and N. Bonod, Phys. Rev. B 84, 125420 (2011).

[7] V. V. Klimov and D. V. Guzatov, Appl. Phys. A 89, 305 (2007).

[8] W. Rechberger, A. Hohenau, A. Leitner, J. R. Krenn, B. Lamprecht, and F. R. Aussenegg, Opt. Commun. 220, 137 (2003).

[9] P. Nordlander, C. Oubre, E. Prodan, K. Li, and M. I. Stockman, Nano Lett. 4, 899 (2004).

[10] R. Ruppin, Phys. Rev. B 26, 3440 (1982).

[11] I. Olivares, R. Rojas, and F. Claro, Phys. Rev. B 35, 2453 (1987).

[12] M. Schmeits and L. Dambly, Phys. Rev. B 44, 12706 (1991).

[13] P. K. Jain, W. Huang, and M. A. El-Sayed, Nano Lett. 7, 2080 (2007).

[14] P. Albella, M. A. Poyli, M. K. Schmidt, S. A. Maier, F. Moreno, J. J. Sáenz, and J. Aizpurua, J. Phys. Chem. C 117, 13573 (2013).

[15] E. Prodan, C. Radloff, N. J. Halas, and P. Nordlander, Science 302, 419 (2003).

[16] P. R. Wiecha, L.-J. Black, Y. Wang, V. Paillard, C. Girard, O. L. Muskens, and A. Arbouet, Sci. Rep. 7, 40906 (2017).

[17] A. Cecconello, L. V. Besteiro, A. O. Govorov, and I. Willner, Nat. Rev. Mater. 2, 17039 (2017).

[18] M. Sun, L. Xu, J. H. Banhg, H. Kuang, S. Alben, N. A. Kotov, and C. Xu, Nat. Commun. 8, 1847 (2017).

[19] E. Kuntman, M. A. Kuntman, J. Sancho-Parramon, and O. Arteaga, Phys. Rev. A 95, 063819 (2017).
[20] E. Kuntman, M. Ali Kuntman, and O. Arteaga, J. Opt. Soc. Am. A 34, 80 (2016).

[21] O. Arteaga and A. Canillas, Opt. Lett. 35, 559 (2010).

[22] F. Capolino, Theory and Phenomena of Metamaterials, edited by F. Capolino (CRC Press, Boca Raton, FL, 2009).

[23] V. V. Gozhenko, L. G. Grechko, and K. W. Whites, Phys. Rev. B 68, 125422 (2003).

[24] A. Pinchuk and G. Schatz, Nanotechnology 16, 2209 (2005).

[25] A. E. Miroshnichenko, S. Flach, and Y. S. Kivshar, Rev. Mod. Phys. 82, 2257 (2010).

[26] K. C. Woo, L. Shao, H. Chen, Y. Liang, J. Wang, and H.-Q. Lin, ACS Nano 5, 5976 (2011).

[27] D. Lin and J.-S. Huang, Opt. Express 22, 7434 (2014).

[28] J. Wu, X. Lu, Q. Zhu, J. Zhao, Q. Shen, L. Zhan, and W. Ni, Nano-Micro Lett. 6, 372 (2014).

[29] L.-Y. Wang, K. W. Smith, S. Dominguez-Medina, N. Moody, J. M. Olson, H. Zhang, W.-S. Chang, N. Kotov, and S. Link, ACS Photonics 2, 1602 (2015).

[30] B. Auguié, J. L. Alonso-Gómez, A. Guerrero-Martínez, and L. M. Liz-Marzán, J. Phys. Chem. Lett. 2, 846 (2011).

[31] X. Yin, M. Schäferling, B. Metzger, and H. Giessen, Nano Lett. 13, 6238 (2013).

[32] W. Kuhn, Zeitschr. Physikal. Chem. 4B, 14 (1929).

[33] R. Ossikovski, O. Arteaga, S. H. Yoo, E. Garcia-Caurel, and K. Hingerl, Opt. Lett. 42, 4740 (2017).

[34] U. Hohenester and A. Trügler, Comput. Phys. Commun. 183, 370 (2012).

[35] F. J. G. de Abajo and A. Howie, Phys. Rev. B 65, 115418 (2002).

[36] K. Claborn, C. Isborn, W. Kaminsky, and B. Kahr, Angew. Chem., Int. Ed. 47, 5706 (2008).

[37] C. Isborn, K. Claborn, and B. Kahr, J. Phys. Chem. A 111, 7800 (2007).

[38] O. Arteaga, Opt. Lett. 40, 4277 (2015). 
[39] T. W. H. Oates, T. Shaykhutdinov, T. Wagner, A. Furchner, and K. Hinrichs, Opt. Mater. Express 4, 2646 (2014).

[40] T. W. H. Oates, T. Shaykhutdinov, T. Wagner, A. Furchner, and K. Hinrichs, Adv. Mater. 26, 7197 (2014).

[41] J. R. Taylor, Classical Mechanics (University Science Books, Herndon, VA, 2005).

[42] M. Schäferling, Chiral nanophotonics: chiral optical properties of plasmonic systems (Springer, New York, 2017).

[43] B. Khlebtsov, A. Melnikov, V. Zharov, and N. Khlebtsov, Nanotechnology 17, 1437 (2006).

[44] J. P. Kottmann and O. J. F. Martin, Opt. Lett. 26, 1096 (2001).

[45] J. Sancho-Parramon, Opt. Lett. 36, 3527 (2011).

[46] F. Hao, Y. Sonnefraud, P. V. Dorpe, S. A. Maier, N. J. Halas, and P. Nordlander, Nano Lett. 8, 3983 (2008).
[47] B. Luk'yanchuk, N. I. Zheludev, S. A. Maier, N. J. Halas, P. Nordlander, H. Giessen, and C. T. Chong, Nat. Mater. 9, 707 (2010).

[48] O. Arteaga, B. M. Maoz, S. Nichols, G. Markovich, and B. Kahr, Opt. Express 22, 13719 (2014).

[49] O. Arteaga, J. Sancho-Parramon, S. Nichols, B. M. Maoz, A. Canillas, S. Bosch, G. Markovich, and B. Kahr, Opt. Express 24, 2242 (2016).

[50] S. Chandel, J. Soni, S. K. Ray, A. Das, A. Ghosh, S. Raj, and N. Ghosh, Sci. Rep. 6, 26466 (2016).

[51] S. Kadkhodazadeh, J. R. de Lasson, M. Beleggia, H. Kneipp, J. B. Wagner, and K. Kneipp, J. Phys. Chem. C 118, 5478 (2014).

[52] J. I. L. Chen, Y. Chen, and D. S. Ginger, J. Am. Chem. Soc. 132, 9600 (2010). 